\title{
The neural bases of resilient semantic system: evidence of variable neuro-displacement in cognitive systems
}

\author{
Je Young Jung ${ }^{1}\left(\mathbb{0} \cdot\right.$ Grace E. Rice $^{2} \cdot$ Matthew A. Lambon Ralph ${ }^{2}$
}

Received: 1 September 2020 / Accepted: 2 April 2021 / Published online: 20 April 2021

(c) The Author(s) 2021

\begin{abstract}
The purpose of this study was to explore an important research goal in cognitive and clinical neuroscience: What are the neurocomputational mechanisms that make cognitive systems "well engineered" and thus resilient across a range of performance demands and to mild levels of perturbation or even damage? A new hypothesis called 'variable neuro-displacement' suggests that cognitive systems are formed with dynamic, spare processing capacity, which balances energy consumption against performance requirements and can be resilient to changes in performance demands. Here, we tested this hypothesis by investigating the neural dynamics of the semantic system by manipulating performance demand. The performance demand was manipulated with two levels of task difficulty (easy vs. hard) in two different ways (stimulus type and response timing). We found that the demanding semantic processing increased regional activity in both the domain-specific semantic representational system (anterior temporal lobe) and the parallel executive control networks (prefrontal, posterior temporal, and parietal regions). Functional connectivity between these regions was also increased during demanding semantic processing and these increases were related to better semantic task performance. Our results suggest that semantic cognition is made resilient by flexible, dynamic changes including increased regional activity and functional connectivity across both domainspecific and domain-general systems. It reveals the intrinsic resilience-related mechanisms of semantic cognition, mimicking alterations caused by perturbation or brain damage. Our findings provide a strong implication that the intrinsic mechanisms of a well-engineered semantic system might be attributed to the compensatory functional alterations in the impaired brain.
\end{abstract}

Keywords Resilient cognitive systems $\cdot$ Neural variable displacement $\cdot$ Semantic cognition $\cdot$ Bilateral representation system $\cdot$ Functional connectivity

\section{Introduction}

One critical feature of any well-engineered system is its resilience to variable performance demands, perturbation and minor damage. How resilience is achieved in higher cognitive systems is important both for cognitive and clinical neuroscience. Although rarely considered in laboratorybased explorations of higher cognition (beyond executive function where demand variations are inherently important),

Je Young Jung

jeyoung.jung@nottingham.ac.uk

$\triangle$ Matthew A. Lambon Ralph

matt.lambon-ralph@mrc-cbu.cam.ac.uk

1 School of Psychology, University of Nottingham, University Park, Nottingham NG7 2RD, UK

2 MRC Cognition and Brain Science Unit (CBU), University of Cambridge, Cambridge CB2 7EF, UK in everyday life we are faced with and are resilient to variations in task difficulty, degraded stimuli, etc. Likewise, after partial brain damage or perturbation, participants can sometimes show impressive resilience and recovery. The current study investigated the mechanisms that support resilience in the domain of semantic cognition.

Recently, we have proposed a new fundamental principle regarding the resilient mechanisms in the brain-'variable neuro-displacement' (Stefaniak et al. 2020). A core tenet in engineering is to design a system that is resilient to functional stresses, as well as to balance performance and energy costs. It seems likely that neurocognitive systems are also designed: (a) to be tolerant to variable levels of performance demand; and (b) to titrate performance against metabolic energy demands. Accordingly, under standard levels of performance demand the full neural system will be downregulated to save energy but have spare capacity that can be utilised when the situation necessitates it (Stefaniak et al. 
2020), known as 'variable displacement' in modern designs of combustion engines (Manring and Johnson 1996). This principle is consistent with the findings that stroke-induced damage can lead to increased neural activation in the perilesional area and/or remote regions, and that this increased activity is correlated with patients' cognitive performance after stroke (Saur et al. 2006; van Oers et al. 2010; Heiss et al. 1999; Szaflarski et al. 2013; Warburton et al. 1999; Murray 2012; Woolgar et al. 2013). Here, we tested this principle focusing on the semantic system by manipulating performance demand.

Semantic cognition allows us to use, manipulate and generalize knowledge. This is a crucial function for communication (verbal and nonverbal) and activities of daily living (e.g., object use) (Lambon Ralph et al. 2017). It can be decomposed into two components: semantic representation - the long-term representation of concepts/semantic memory and semantic control-mechanisms to generate time- and context-appropriate semantic behaviours (Jefferies 2013). The neural basis of semantic cognition reflects a large-scale network across the frontal, temporal and parietal cortex (Patterson et al. 2007; Binder et al. 2009). Convergent evidence suggests that the ventrolateral anterior temporal lobe (ATL) is the centre-point of a transmodal hub that supports semantic representation (Binney et al. 2010; Lambon Ralph 2014; Peelen and Caramazza 2012; Coccia et al. 2004; Chan et al. 2001), whereas prefrontal and temporoparietal cortices (e.g., inferior frontal gyrus; IFG, posterior middle temporal gyrus; pMTG, and inferior parietal lobe; IPL) are involved in controlled retrieval of semantic knowledge (Noonan et al. 2013; Thompson-Schill et al. 1997; Badre and Wagner 2002; Jefferies and Lambon Ralph 2006).

There are two existing sources of evidence for resilience in the semantic system. The first comes from patients with unilateral ATL resection/damage (either left or right) who exhibit mild semantic impairments (reflected in slower response times or reduced accuracy on demanding semantic tasks) but perform much better overall than patients with bilateral ATL resection/damage (Lambon Ralph et al. 2010a, 2012; Bi et al. 2011). The same pattern was shown in the seminal investigations of unilateral versus bilateral ATL resections in non-human primates (Brown 1888; Kluver and Bucy 1939) and one human case (Terzian and Ore 1955), in which unilateral resection resulted in transient multimodal associative agnosia, whereas bilateral resection caused severe, chronic deficits. Whilst these studies show that the semantic system is somewhat robust against partial damage (Schapiro et al. 2013; Lambon Ralph et al. 2010a), it remains unclear what neural mechanisms underlie this resilience.

The second line of evidence comes from recent studies that combined fMRI and transcranial magnetic stimulation (Binney and Lambon Ralph 2015; Jung and Lambon Ralph 2016). Using a "perturb-and-measure" approach (Paus
2005), inhibitory repetitive TMS (rTMS) was delivered over the left ATL and the resultant behavioural and neural changes were measured. rTMS over the left ATL decreased regional activity in the target site yet increased activity at the contralateral ATL. This upregulation in the right ATL contributed to residual semantic performance (stronger activity was associated with faster reaction times). Furthermore, an effective connectivity analysis revealed that, after the inhibitory rTMS over the ATL, there was increased connectivity from the right ATL to the left ATL (Jung and Lambon Ralph 2016).

As well as resultant changes within the ATL-based representational system, functional neuroimaging and patients' studies indicate that there is also potentially important upregulation of the semantic control regions. Patients with temporal lobe epilepsy can show upregulation in the IFG during semantic tasks before (Billingsley et al. 2001) and after the ATL resection (Noppeney et al. 2005). fMRI studies have reported increased IFG and pMTG activity during demanding semantic processing in healthy individuals (Thompson-Schill et al. 1997; Wagner et al. 2001; Noppeney et al. 2004; Badre et al. 2005). Furthermore, the distributed network for semantic control, itself, also seems to exhibit dynamic, resilient-related changes in that rTMS to IFG generates compensatory increased activation in the strongly connected pMTG (Jung et al. 2016; Nee et al. 2013; Spreng et al. 2013). These studies suggest that the resilience of the semantic system can be attributed to the involvement of semantic control regions.

The limited data on this topic to date principally centre on patient or TMS investigations-i.e., examining the brain after damage or perturbation. The central aim of this study was to test whether these dynamic changes (e.g., alterations in the regional activity and functional connectivity) are specific to the impaired brain (indicating compensatory changes that are triggered by brain damage/perturbation) or whether they reflect intrinsic mechanisms of a well-engineered cognitive system. Two previous studies, with a modest sample size $(N<15)$, examined the neural dynamics of object recognition by manipulating semantic demands and reported increased connectivity between the ATL and fusiform gyrus (Clarke et al. 2011; Poch et al. 2015). Here, we tested intrinsic mechanisms of a well-engineered cognitive system with a larger sample size $(N=41)$ by investigating the neural dynamics of semantic system in healthy participants at different levels of performance challenge. To ensure generalisation across different types of challenge and stimulus modality, we manipulated task difficulty in two different ways (stimulus difficulty vs. response timing) and, in the response timing experiment, compared the results for verbal (written words) and nonverbal (picture) semantic processing. If the changes observed in previous studies of patients or rTMS reflect intrinsic features of a well-engineered system 
then we expected to see the same types of dynamic changes in the healthy system under performance pressure. These would include changes in the ATL semantic representational system and the IFG-pMTG semantic and domain-general executive control networks.

\section{Materials and methods}

\section{Participants}

Forty-one healthy participants were recruited for the study. Twenty-one participants $(7$ females, mean age, $22 \pm 3.1$ years, education: 16.2 years) participated in the stimulus manipulation (SM) experiment and 20 participants (9 females, mean age, $38 \pm 12.2$ years, education: 17.1 years) in the response timing manipulation (RM) experiment. All participants were right-handed, native English speakers who had normal or corrected-to-normal vision. Participants who have contraindications to MRI or any neurological, psychiatric, or relevant medical condition were excluded. They received a detailed explanation of the study and gave written informed consent prior to the experiment. The experiment was approved by the local ethics committee.

\section{Experimental design}

Participants completed two tasks with two different level of difficulty (easy vs. hard) during fMRI: a semantic task and a matched control task. We manipulated task difficulty in two different ways: stimulus manipulation (SM) and response time manipulation (RM) (Fig. 1). The RM data have been reported in a recent examination of the semantic network in patients with ATL resection (Rice et al. 2018).

In the SM experiment, participants performed a category judgement task. The stimuli for the easy condition were from the Levels of Familiarity, Typicality, and Specificity (LOFTS) semantic battery (Rogers et al. 2015). The concrete words covered a variety of categories, including animals, vehicles, tools, foods, and plants. The stimuli for the hard condition were selected from a previous study rating abstract nouns according to conceptual features (i.e., emotion, sensation, action, thought, time, quantity, morality, social interaction, and teaching) (O'Reilly et al. 2012). 335 abstract nouns were selected and six native English speakers rated them for their category. In this study, we used abstract nouns that all six raters agreed to a category. Participants were asked to indicate which of two categories was appropriate for a target. In each trial, three words were presented on the screen, a target on the top and two choices at the bottom (Fig. 1a). A pattern matching task was used as a control task. The items for the control task were generated by visually scrambling items from the semantic task. Each pattern was created by scrambling each item into 120 pieces and re-arranging them in a random order. Participants were asked to select which of two patterns was identical to a target pattern (Fig. 1c left). In the hard condition, the patterns for a right answer were $180^{\circ}$ rotated (Fig. 1c right).

In the RM experiment, participants performed the Camel and Cactus test (CCT) (Bozeat et al. 2000) and an occupation matching task for famous people as semantic tasks (Rice et al. 2018) (Fig. 1b). To investigate the effects of modality, both tasks were presented either as written words and pictures. On each trial, three items were presented on the screen, a probe concept at the top and two choices at the bottom. Participants were asked to decide which of the two alternatives was semantically related to the target for the CCT and to indicate which of two alternatives had the same occupation for the people matching task. This resulted in four semantic conditions (CCT [word], CCT [picture], Famous Faces, Famous Names). In the occupation matching task, all items shared the same gender. The same pattern matching task was used as a control task (Fig. 1c left). To manipulate the difficulty in response, the stimuli were presented with $2.5 \mathrm{~s}$ response window for the hard condition and $5 \mathrm{~s}$ for the easy condition. All participants were informed that they would perform the tasks with different response time window.

\section{Experimental procedures}

The total scan time of the SM experiment was about 10 min. During scanning, stimuli were presented in a block design and each block contained four trials from one experimental condition (semantic: concrete and abstract words and control: patterns and rotated patterns). Each stimulus and the response screen were presented for $5000 \mathrm{~ms}$, with an interstimulus interval of $500 \mathrm{~ms}$. The four experimental conditions were sampled six times in a counterbalanced order, giving a total of 24 blocks.

The total scan time of the RM experiment was about $8.45 \mathrm{~min}$ for the easy condition and $4.2 \mathrm{~min}$ for the hard condition. During scanning, stimuli were presented in a block design and each block contained three trials from one experimental condition. Participants performed eight functional scans and each scan contained a semantic and a matched control condition. For the easy functional scans (four scans), the stimuli (semantic: CCT [word], CCT [picture], famous names or famous faces; control: scrambled pictures or scrambled words) were presented for $5000 \mathrm{~ms}$, with an inter-stimulus interval of $500 \mathrm{~ms}$. For the hard scans (four scans), difficulty was increased by presenting stimuli twice as quickly, at $2500 \mathrm{~ms}$. The functional scans were interleaved to avoid any habituation to the speed of presentation.

All participants completed practice trials before beginning the scan to familiarize them with the tasks. E-prime 


\section{A Stimulus manipulation (SM): concrete vs. abstract word categorization}
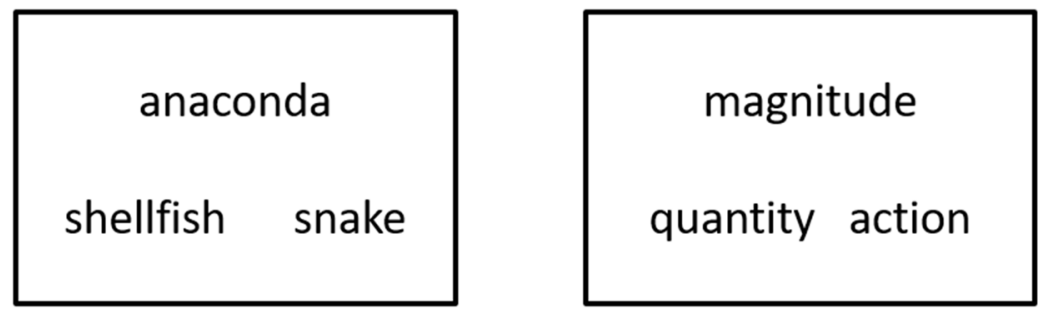

\section{B Response time manipulation (RM): $5 s$ vs. 2.5 s response time window}
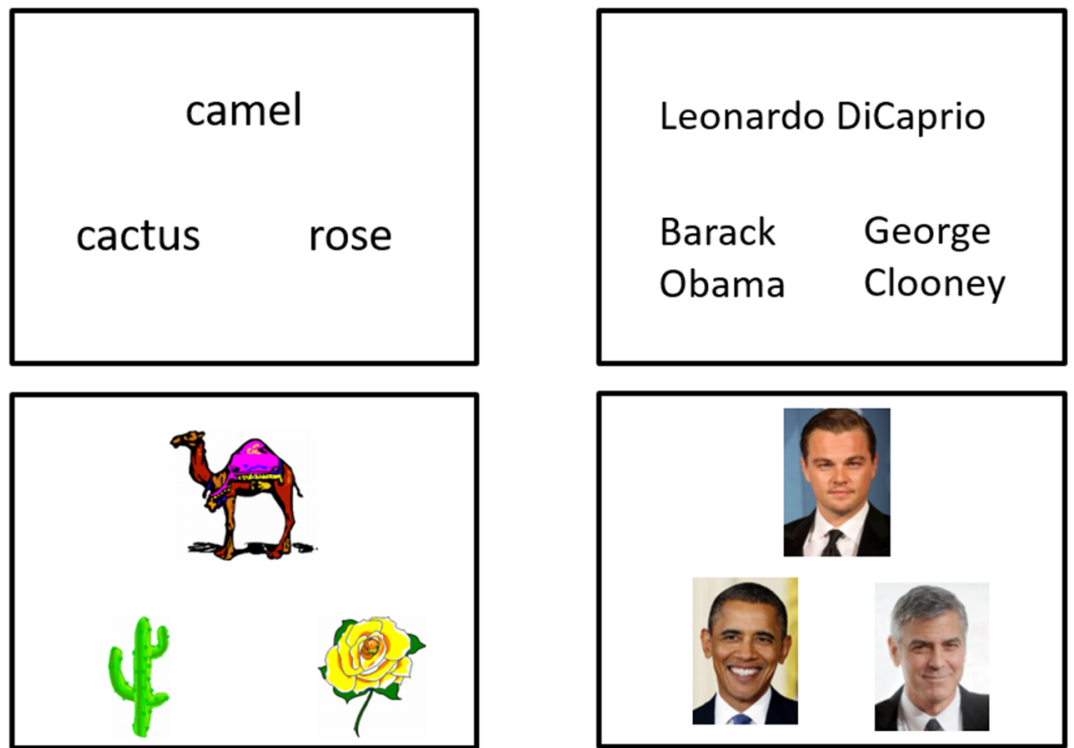

\section{Control task (pattern matching): Upright vs. $180^{\circ}$ rotated (only for SM)}
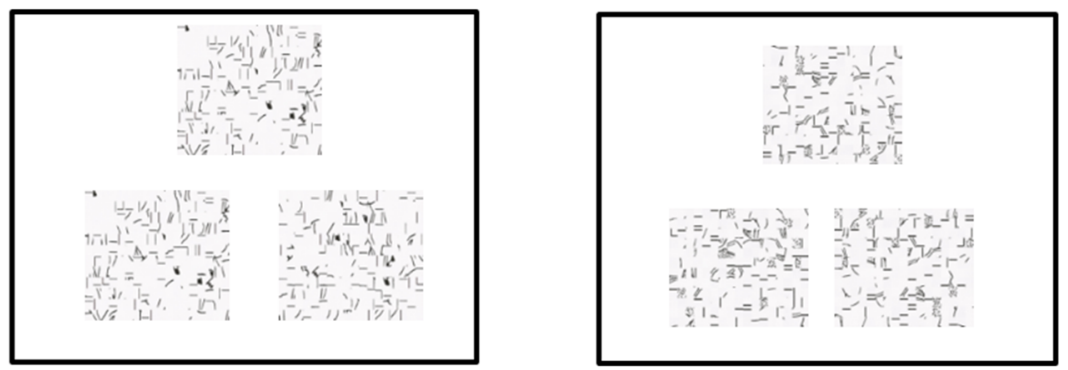

Fig. 1 Experimental design. a Stimulus manipulation (SM). b Response time manipulation (RM). c Control task (pattern matching)

software (Psychology Software Tools Inc., Pittsburgh, PA, USA) was used to display stimuli and to record responses.

\section{fMRI data acquisition and analysis}

Imaging was performed on a 3T Philips Achieva scanner using a 32-channel head coil with a SENSE factor of 2.5.
To improve signal-to-noise (SNR) in the ATL, we utilised a dual-echo fMRI protocol developed by Halai et al. (2014). The fMRI sequence included 42 slices, $96 \times 96$ matrix, $240 \times 240 \times 126 \mathrm{~mm} \mathrm{FOV}$, in-plane resolution $2.5 \times 2.5$, slice thickness $3 \mathrm{~mm}, \mathrm{TR}=2.8 \mathrm{~s}, \mathrm{TE}=12 \mathrm{~ms}$ and $35 \mathrm{~ms}$. All images were acquired using a tilt, up to $45^{\circ}$ off the AC-PC line, to reduce ghosting artefacts in the temporal lobes. 
In the SM experiment, 190 dynamic scans were acquired and, in the RS experiment, 177 dynamic scans for the easy condition and 88 dynamic scans for the hard condition (all functional scans included two dummy scans, which were excluded). The structural image was acquired using a 3D MPRAGE pulse sequence with 200 slices, in planed resolution $0.94 \times 0.94$, slice thinkness $0.9 \mathrm{~mm}, \mathrm{TR}=8.4 \mathrm{~ms}$, and $\mathrm{TE}=3.9 \mathrm{~ms}$.

Analysis was carried out using SPM8 (Wellcome Department of Imaging Neuroscience, London; www.fil.ion.ucl. ac.uk/spm). The dual gradient echo images were extracted and averaged using in-house MATLAB code developed by Halai et al (2014). Functional images were realigned correcting for motion artefacts and different signal acquisition times by shifting the signal measured in each slice relative to the acquisition of the middle slice prior to combining the short and long echo images. The mean functional EPI image was co-registered to the individual T1-weighted image and segmented using the DARTEL (diffeomorphic anatomical registration through an exponentiated lie algebra) toolbox (Ashburner 2007). Then, normalization was performed using DARTEL to warp and reslice images into MNI space and smoothing was applied with an $8 \mathrm{~mm}$ full-width half-maximum Gaussian filter.

At the individual subject level, contrasts of interest were modelled using a box-car function convolved with the canonical hemodynamic response function. For the SM experiment, four separate regressors were modelled according to task and difficulty (semantic: concrete and abstract words and control: patterns and rotated patterns). For the RM experiment, two separate regressors were modelled in each functional scan: (1) semantic condition (either: CCT [picture], CCT [word], Famous Faces, Famous Names) and (2) control condition (either scrambled pictures or scrambled words).

At the group level, a two-factorial ANOVA with task (semantic vs. control) and difficulty (easy vs. hard) was conducted for the main effect of task and interaction between task and difficulty and T-contrasts were established for the contrast of semantic $>$ control and hard $>$ easy across the experiments. The same analyses were performed according to the modality (words and pictures) and difficulty manipulation (SM and RM). To identify activation maps from each task condition, we used a cluster-based inference method. Whole-brain maps were thresholded at $p<0.001$ at the voxel level, with a FWE-corrected cluster threshold of $p<0.05$, $k>100$.

\section{Region of interest (ROI) analysis}

An a priori ROI analysis was employed to assess the level of activation in semantic regions including the vATL, IFG, and pMTG. Peak coordinates were taken from previous studies investigating the semantic system: vATL [MNI: $-36-15$ -30; $36-15-30$ ] (Binney et al. 2010), IFG (BA 45: p. Tri) [MNI: -45 19 18; 4723 26] (Noonan et al. 2013), IFG (BA47: p. Orb) [MNI: -45 $27-15 ; 4527-15$ ] (Deco et al. 2015), and pMTG [MNI: -66 -42 3; $66-42$ 3] (Binney et al. 2010). The right hemisphere ROIs were created using the homologous coordinates. Each ROI was created as a sphere with $8 \mathrm{~mm}$ radius.

\section{Functional connectivity (FC) analysis}

To investigate changes in vATL functional connectivity to the rest of the brain, we conducted psychophysiological interaction analysis (PPI). The vATL has been reported as the centre-point of a representational hub, which interacts with other semantic control regions and spokes to generate coherent semantic knowledge (Lambon Ralph et al. 2010b, 2017; Lambon Ralph 2014). The PPI analysis describes neural responses in one brain area in terms of the interaction between influences of other brain regions and a cognitive process (Goni et al. 2014). The PPI analysis employed a design matrix with three regressors: (i) the "psychological variable" representing the cognitive process of interest; (ii) the "physiological variable" representing the neural response in the seed region and (iii) the interaction term of (i) and (ii). To quantify the physiological variable, we extracted the individual time series from the left vATL (lvATL) as a seed region. For the lvATL seed, the time courses were de-convolved based on the model for the canonical hemodynamic response to construct a time series of neural activity, which was the physiological factor. Interaction terms were calculated separately for each experimental condition (semantic: easy vs. hard), as the product between the vector of the condition and the physiological factor. The PPI terms were also convolved with the hemodynamic response function. Then, we revealed how the hard semantic condition induced functional connectivity change to the corresponding seed region compared with the easy condition. As the PPI is very stringent (O'Reilly et al. 2012), we used the significance threshold at $p<0.005$ at the voxel level, with a FWE-corrected cluster threshold of $p<0.05, k>30$.

To investigate the difficulty effects on the semantic processing between semantic regions, we employed the Functional Connectivity (CONN) Toolbox (http://web.mit.edu/ $\mathrm{swg} /$ software.htm). This method enables examination of network interactions during each condition of an fMRI task and thus comparison of FC between different conditions (semantic: easy vs. hard). Pre-processed images were entered to the toolbox. Data were filtered using a band pass filter $(0.01<f<2)$ to decrease the effect of low-frequency drift. White matter, cerebrospinal fluid, and physiological noise source reduction were taken as confounds, following the implemented CompCor strategy (Behzadi et al. 2007). 
Head motion was taken into account and rotational and translational motion parameters and their first-order temporal derivatives were regressed out. The onset and duration of each experimental condition was supplied to the toolbox so as to extract the connectivity generated for easy and hard semantic processing. The key regions of the semantic network were included in this analysis (vATL, IFG [p.Orb], IFG [p. Tri], and pMTG) and bivariate correlations were calculated between each pair of ROIs as reflections of connections according to the experimental conditions. Planned paired $t$ tests were performed on the FC between ROIs for the easy and hard semantic conditions $(p<0.05)$.

\section{Results}

\section{Behavioural results}

A repeated-measures ANOVA with task (semantic vs. control) and difficulty (easy vs. hard) was conducted for each experimental dataset (SM and RM) and the combined dataset $(\mathrm{SM}+\mathrm{RM})$. In accuracy, the SM dataset revealed a significant main effect of difficulty $\left(F_{1,20}=35.10, p<0.001\right)$ and an interaction $\left(F_{1,20}=10.09, p<0.01\right)$. The RM dataset showed a significant main effect of difficulty $\left(F_{1,19}=42.46\right.$, $p<0.001)$. In reaction time (RT), the SM dataset showed a significant main effect of task $\left(F_{1,20}=42.87, p<0.001\right)$, difficulty $\left(F_{1,20}=212.94, p<0.001\right)$ and an interaction $\left(F_{1,20}=22.04, p<0.001\right)$. The RM dataset showed a significant main effect of difficulty $\left(F_{1,19}=199.12, p<0.001\right)$. Post hoc paired $t$ tests revealed that the difficulty manipulation was successful for both datasets and for both tasks (Fig. 2). In the SM dataset, the accuracy was significantly reduced and the RT was increased for the hard condition compared to the easy condition for both semantic and control tasks $(p<0.001)$. In the RM dataset, the accuracy was significantly decreased in the hard condition relative to the easy condition, whereas the RT was faster in the hard condition due to the response time manipulation $(p<0.001)$. The combined dataset also showed a significant main effect

\section{A Semantic task}
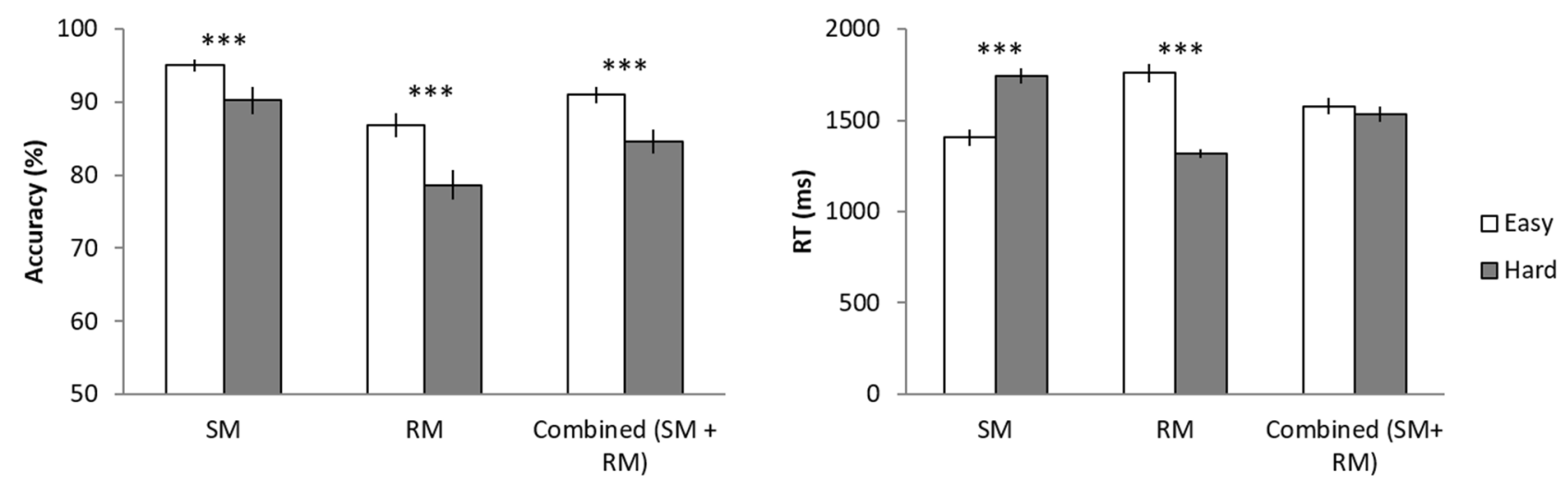

\section{B Control task}
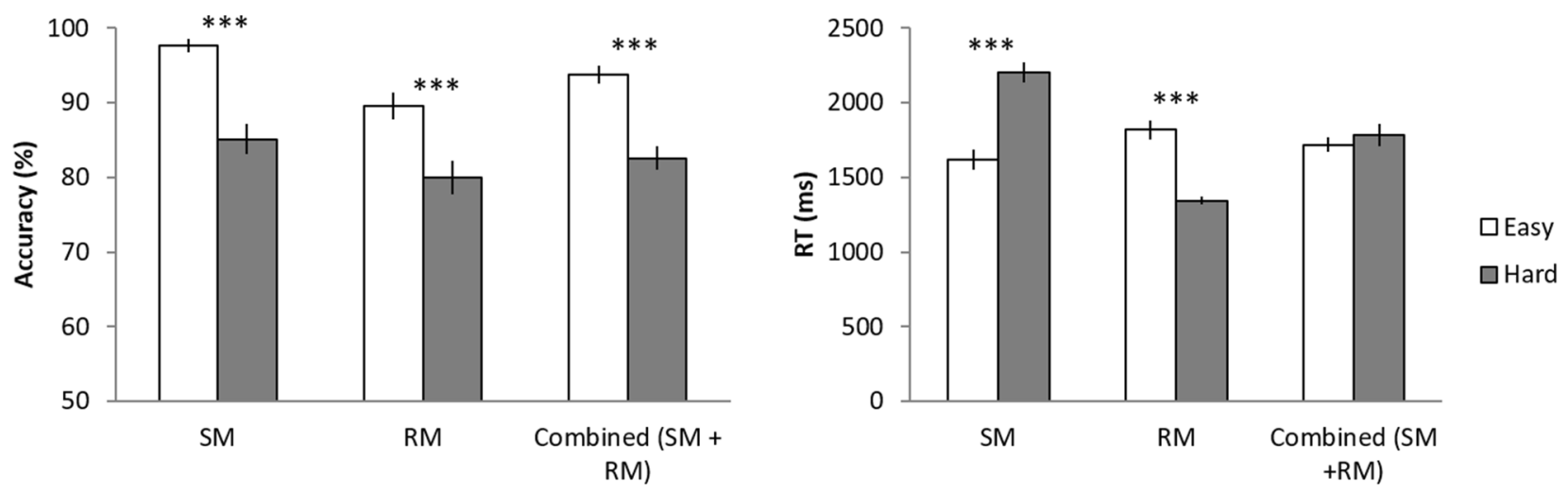

Fig. 2 Behavioural results. a Behavioural results of semantic tasks. b Behavioural results of control tasks. White bars represent the easy condition performance and grey bars the hard condition performance. Error bars indicate standard errors. *** $p<0.001$ 
of difficulty $\left(F_{1,40}=78.63, p<0.001\right)$ and an interaction $\left(F_{1,40}=9.06, p<0.01\right)$ in accuracy. Post hoc paired $t$ tests revealed that accuracy was significantly decreased in the hard condition for both tasks $(p<0.001)$. In addition, participants made slightly fewer errors during the control task than the semantic task in the easy condition $(p<0.005)$. However, the accuracy rates were high for both tasks (semantic: 90\%, control: $93 \%$ ) in the easy condition. In RT, there was a significant main effect of task $\left(F_{1,40}=24.28, p<0.001\right)$ and an interaction between task and difficulty $\left(F_{1,40}=7.78\right.$, $p<0.001)$. For the RT in the combined data, we did not perform post hoc analysis due to the experimentally manipulated response time. The other comparisons did not reach the significance level $(p s>0.05)$. The results of modality (word and picture) are summarised in Fig. S1.

\section{fMRI results}

The whole brain analyses revealed that the semantic task $(\mathrm{SM}+\mathrm{RM})$ evoked significant activation in the left IFG, vATL, pMTG, fusiform gyrus, medial prefrontal cortex (mPFC), superior frontal gyrus (SFG), and precuneus (PCC) regardless of task difficulty (semantic $>$ control) (Fig. 3a top and Table S1). The hard semantic condition induced more widespread activation in the same regions and additional activation in the left angular gyrus (AG) and the right ATL (lateral and ventral), anterior MTG (aMTG), and pMTG. It should be noted that the additional activation evoked by the hard condition was not distinct from the pattern of activity observed in the easy semantic condition if a less stringent threshold was applied $\left(p_{\text {unc }}<0.005\right.$ at a voxel level). Even in the easy semantic condition, we found widespread activation in the bilateral ATL, IFG, pMTG, and AG as well as the left precentral gyrus, hippocampus, and basal ganglia (Fig. 3a bottom and Table S1). The control task (visuospatial processing) activated the bilateral intraparietal sulcus (IPS), inferior parietal lobe (IPL), middle frontal gyrus (MFG), SFG, superior occipital gyrus (SOG), middle occipital gyrus (MOG) and cuneus during the easy condition. The hard control processing was involved in the same regions as well as the right superior parietal lobe (SPL), IFG (pars opercularis), inferior temporal gyrus (ITG), which overlaps with the easy condition activation map at a lower threshold (Fig. S2). Subsequent ROI analyses demonstrated that more demanding semantic processing significantly increased regional activity in the left semantic regions (IFG [p. orb], pMTG, and vATL) compared to the easy semantic condition. In the right hemisphere, only the vATL showed a significant upregulation during the hard semantic processing whereas the other semantic regions in the right hemisphere did not show a difficulty modulation effect (Fig. 3b).

The same analyses were performed on each dataset (SM and RM), split according to modality. The results for the word modality were similar to the main findings; the easy condition evoked significant activation in the left-lateralized semantic system and the hard condition recruited more widespread activation in the left semantic regions as well as the right vATL, aMTG, AG, and mPFC (Fig. S3A-C). In contrast to the word modality, the picture modality evoked bilateral activation in the vATL and pMTG as well as the left IFG for the easy condition and additional activation in the right IFG for the hard condition (Fig. S3D). ROI analyses also showed similar results to the main findings in the word modality: the regional activity in left semantic regions significantly increased in the hard condition and only the right vATL showed significant upregulation (Fig. S4). In contrast, for the picture modality there was no significant upregulation in semantic regions during the more demanding condition. It should be noted that participants performed the picture-based semantic task with lower accuracy and slower RT than the word-based tasks, even in the easy condition (Fig. S1 and Supplementary Results). Therefore, the neural differences found in the picture modality might be driven by picture processing being inherently more demanding than word processing; thus, even in the easy picture semantic task there was recruitment of the right vATL and greater activation in the left semantic regions.

To explore potential differences between the two types of difficulty manipulation, we compared the SM with RM in the hard semantic condition. Semantically demanding SM recruited the MPFC and ACC more than RM, whereas demanding RM evoked increased activity in the left ATL, bilateral IFG, IPS, AG and fusiform gyrus relative to SM (Fig. S5A). In the direct comparison between the easy and hard condition in each dataset, the difficulty effect was found in the left lateral ATL in the SM and the left IFG and ventromedial ATL in the RM (Fig. S5B). There was a significant interaction effect in the bilateral IFG, showing that semantically demanding RM upregulated these regions, whereas hard SM down regulated them (Fig. S5C). These results suggest that the nature of SM is associated with differential involvement of the semantic representation (abstract word processing) (Hoffman et al. 2015), whereas the RM involves both the semantic representation and parallel executive control system.

Several regions showed an interaction between task and difficulty, many of which overlapped with the areas exhibiting the effect of task and/or difficulty (Fig. 4 and Table S2). Specifically, the left IFG and pMTG, as semantic control regions, showed positive activation for the semantic task and greater activation in the hard semantic condition. The interaction in the AG arose from differential deactivation particularly for the hard visuo-spatial processing. As key regions of the default mode network $(\mathrm{DMN})$, the $\mathrm{mPFC}$ and precuneus revealed deactivation for both tasks but a differential difficulty effect was found 


\section{A Semantic > Control}
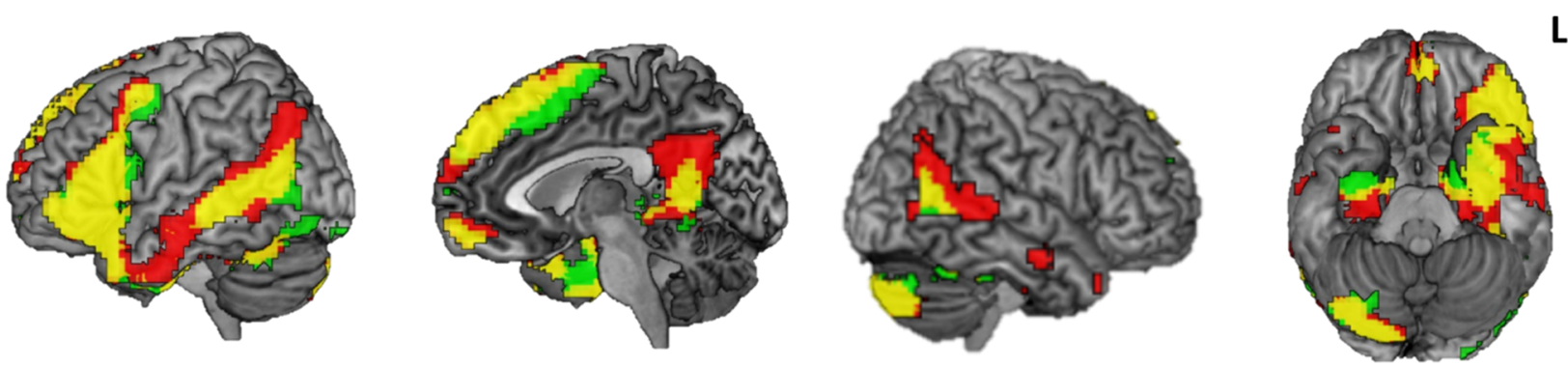

Easy $(p<0.001)$

Hard ( $p<0.001) \square$ Overlapping
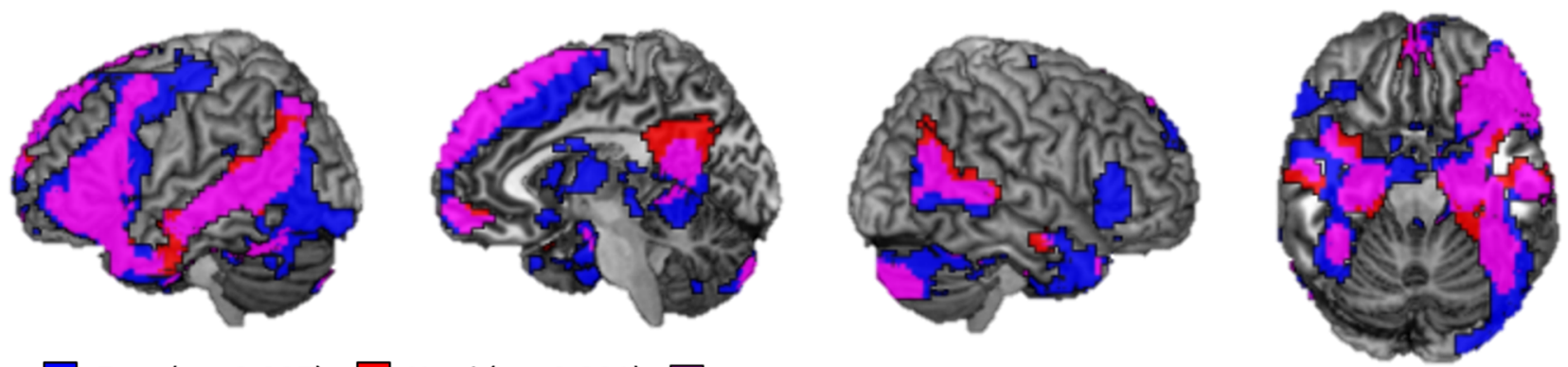

Easy $(p<0.005) \square$ Hard $(p<0.001) \square$ Overlapping

\section{B ROI location}

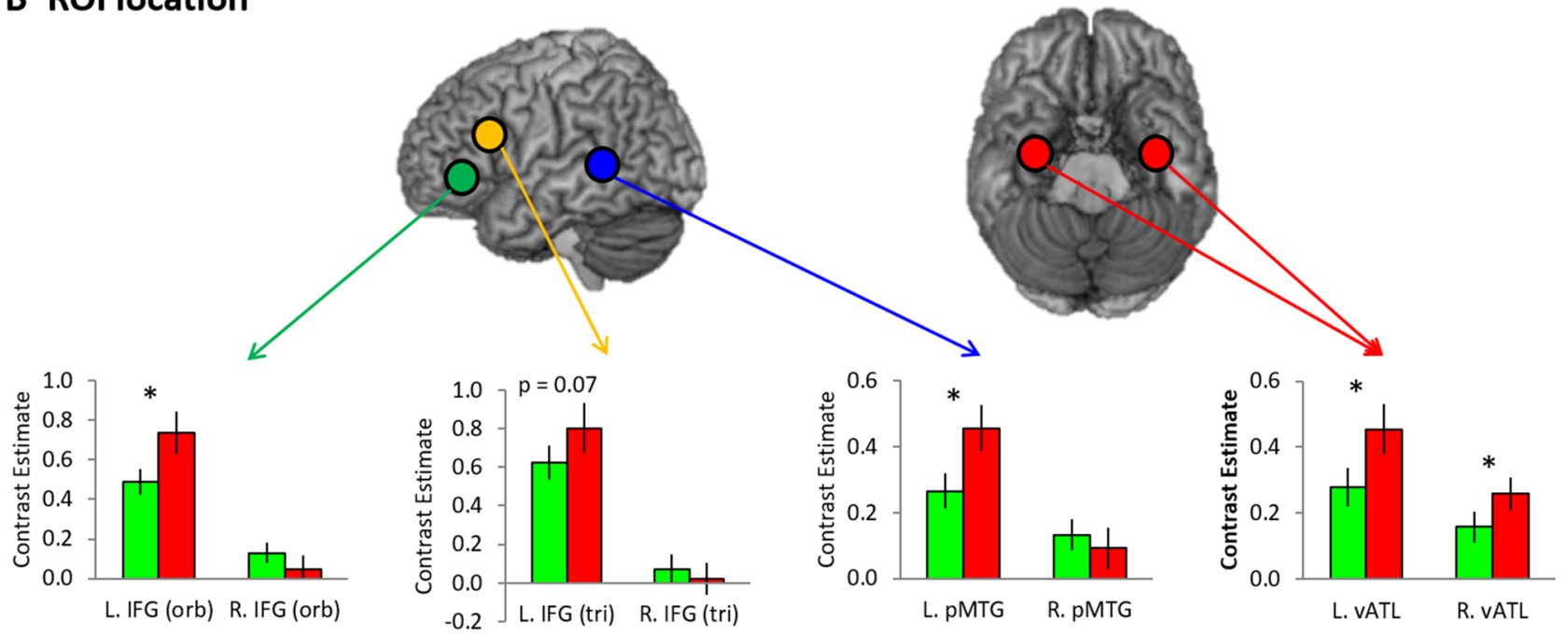

Fig. 3 fMRI results. a Brain activation map of the contrast (Semantic $>$ Control) thresholded at $p_{\text {FWE-corrected }}<0.001$. Green colour indicates the results of the easy semantic condition. Red colour indicates the results of the hard semantic condition. Yellow colour represents overlapping between the easy and hard condition. Blue indicates

in these regions according to tasks. The mPFC showed decreased deactivation for hard semantic processing and increased deactivation for demanding visuospatial processing. The precuneus showed the difficulty effect only for visuospatial processing-more deactivation during the hard control condition. The IPS, a key region of the the result of the easy semantic condition with a lower threshold $\left(p_{\text {FWE-corrected }}<0.005\right)$. Pink represent the overlapping between the hard condition and easy condition with a lower threshold. b ROI results. Green bars represent the easy condition and red bars the hard condition. Error bars indicate standard errors. ${ }^{*} p_{\text {FDR-corrected }}<0.05$

multiple-demanding system (MD) exhibited a task-general effect of difficulty-more activation for demanding condition regardless of tasks. The task-opposite pattern was found in the left lateral occipital cortex (LOC), right ITG and right frontoparietal regions, such that the difficulty effect was observed only for visuospatial processing. 
L. IPS

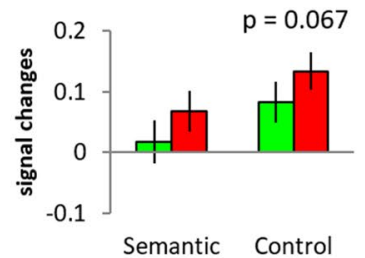

L. IFG

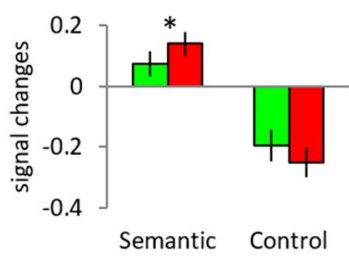

L. PMTG

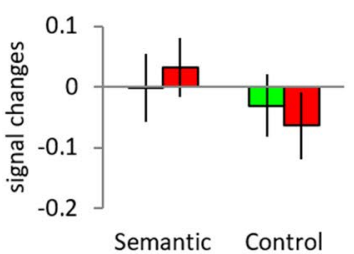

L. AG
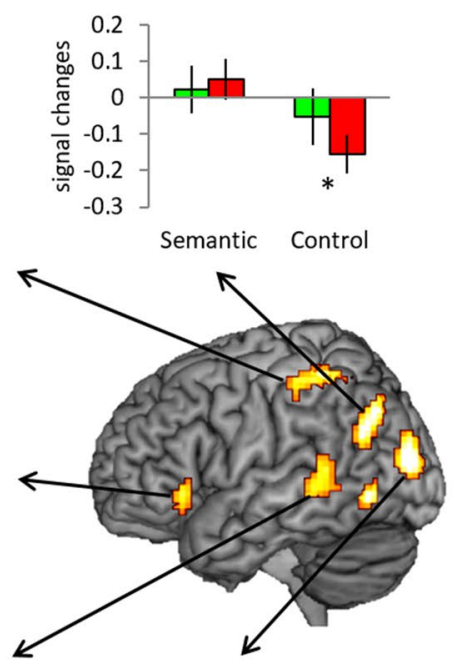

L. LOC

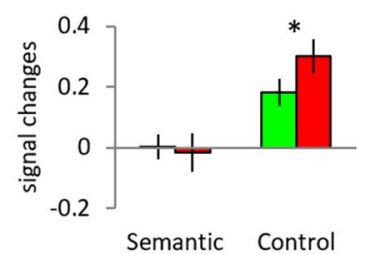

R. SPL
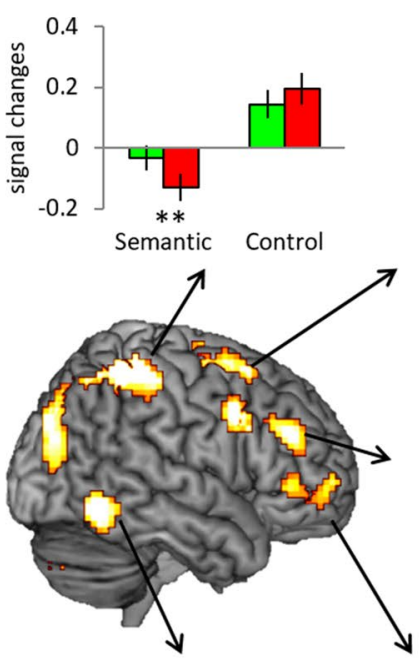

R. ITG

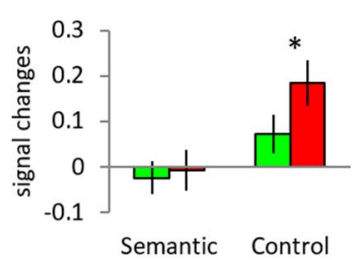

R. SFG

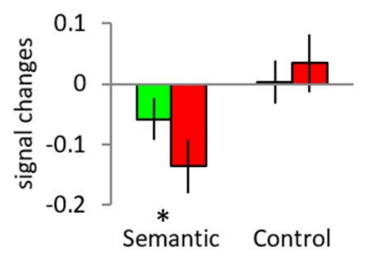

R. MFG

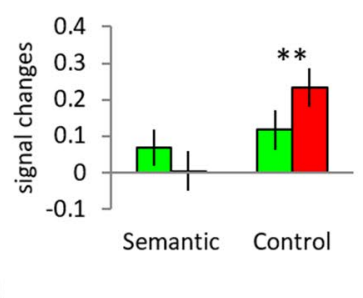

R. IFG

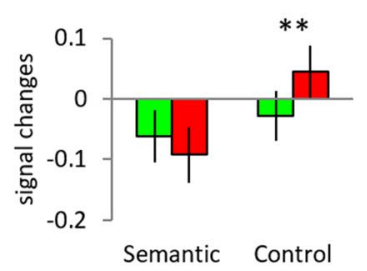

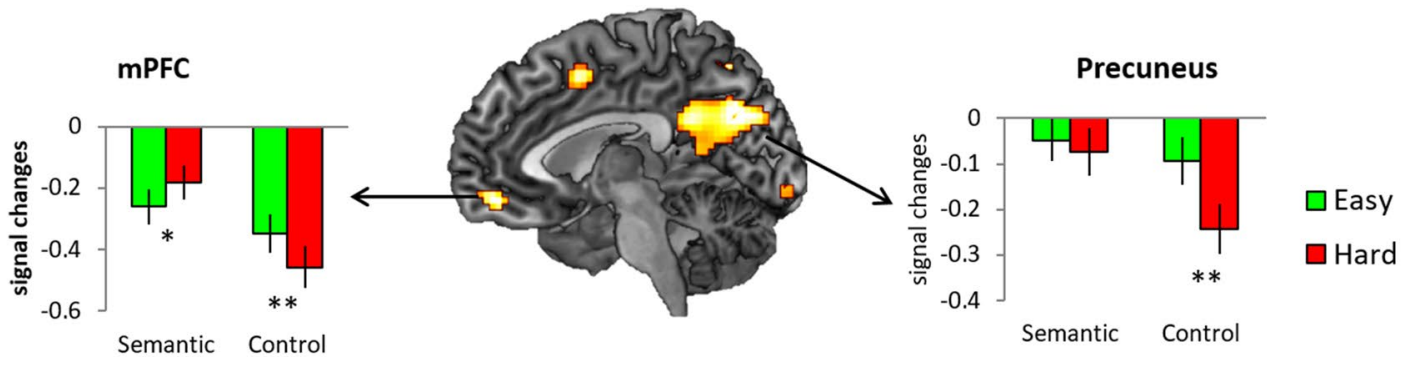

Fig. 4 The interaction between task and difficulty. Green bars represent the easy condition and red bars the hard condition. Error bars indicate standard errors. $* p<0.05$. $* *<0.01$

These same analyses were repeated on each dataset, split according to modality and the results are summarised in the Supplementary Results and Fig. S6. Here, we note that the results of word modality in the SM and RM were similar to the main findings (Fig. S6). However, the interaction effect in the picture modality data revealed activation in the bilateral IFG, temporal pole (TP), left AG, LOC, right SPL, ITG, and mPFC (Fig. 6b). Subsequent ROI analyses demonstrated increased activation in the left AG, the left LOC and right ITG and decreased deactivation in the PCC. These findings indicate that the picture modality engaged regions associated with visuospatial processing.

\section{FC results}

PPI analyses were conducted to examine how vATL connectivity was modulated by difficulty. The easy semantic condition showed that the left vATL was significantly connected with the bilateral IFG, left orbitofrontal cortex (OFC), supplementary motor area (SMA), visual cortex, and cerebellum. The hard semantic condition increased the left vATL connectivity to the same regions found in the easy condition as well as additional areas including the right vATL, left IPS, MFG mPFC, and insula (Fig. 5a).

We examined how ROI-to-ROI connectivity in the semantic network (SN: IFG [p. orb, p. tri], pMTG, and 


\section{A I.ATL connectivity}

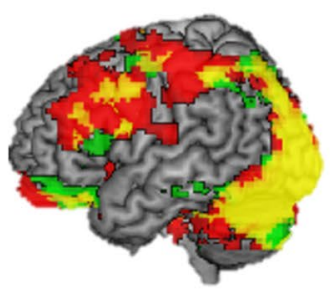

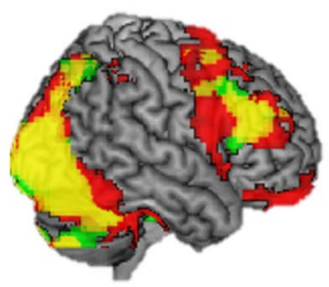

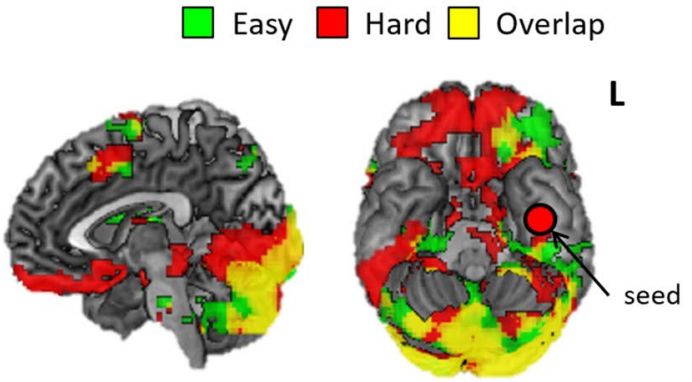

Easy $\square$ Hard $\square$ Overlap

\section{B SN connectivity}
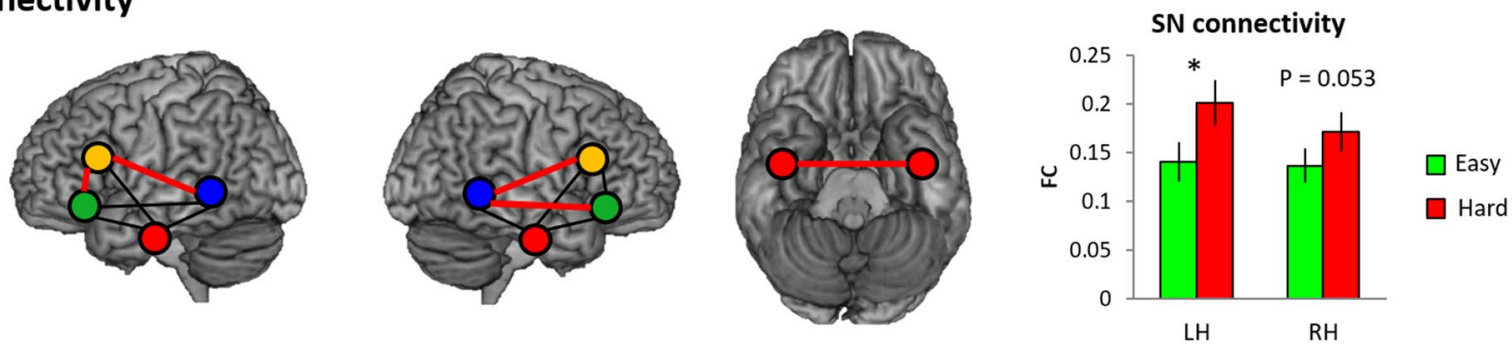

- Connection between ROIs

Increased FC relative to the easy condition, $\mathrm{p}<0.05$

\section{C mPFC connectivity}
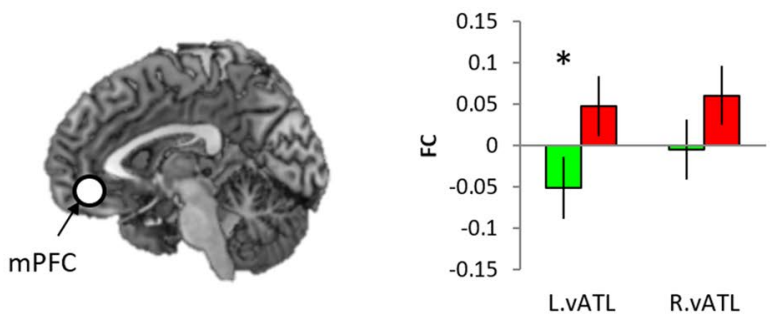

Fig. 5 Functional connectivity (FC) analysis. a The results of PPI with the 1.vATL seed. Green colour indicates the results of the easy semantic condition. Red colour indicates the results of the hard semantic condition. Yellow colour represents overlapping between them. b The results of ROI-to-ROI analysis in the semantic network $(\mathrm{SN})$. Black line represents a connection between ROIs and red line

vATL) changed across the semantic difficulty levels. The FC between the IFG and pMTG in both hemispheres was significantly increased in the hard condition (Fig. 5b). Also, the averaged $\mathrm{FC}$ in all semantic regions (SN connectivity) in the both hemispheres was significantly increased in the hard semantic condition.

ROI and PPI analyses revealed there was greater activation in the mPFC during more demanding semantic processing. We explored the semantic difficulty effect in the FC between the mPFC and semantic regions. A $2 \times 2$ ANOVA with difficulty (easy vs. hard) and hemisphere (left vs. right) was conducted. The results demonstrated that the hard semantic condition significantly increased the mPFC-vATL connectivity (a main effect of difficulty: $F_{1,40}=4.40$, $p<0.05$ ) and the mPFC-right pMTG connectivity (a main

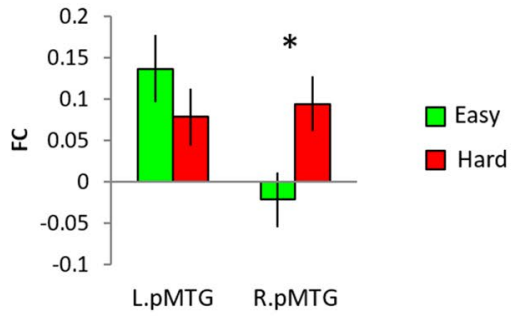

indicates a significantly increased connection between ROIs in the hard semantic condition compared to the easy semantic condition. c The results of the mPFC connectivity to semantic regions. Green bar indicates the easy semantic condition. Red bar indicates the hard semantic condition. $* p<0.05$

effect of hemisphere: $F_{1,40}=6.41, p<0.05$, interaction: $F_{1,40}=4.07, p<0.05$ ) (Fig. 5c). Post hoc paired $t$-tests confirmed the findings. The same analyses were performed in each dataset, split according to modality, and the results are summarised in Fig. S7.

\section{FC-behavioural correlations}

We correlated semantic performance with FC between semantic regions to examine which functional connectivity aligns with the changing semantic demand. Accuracy during the easy semantic condition was positively correlated with the left-right vATL and left vATL-left IFG [p. Tri] connectivity (Fig. 6a). RT in the easy semantic condition was significantly correlated with the left-right 


\section{A Easy semantic condition}

I.vATL-r.vATL

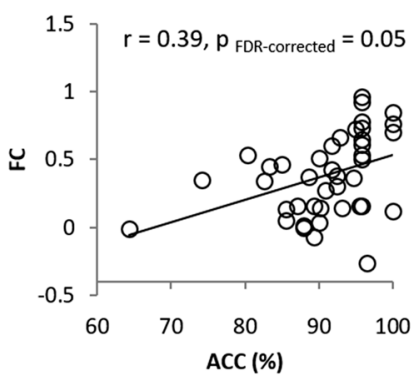

\section{I.VATL-I.IFG (p.Tri)}

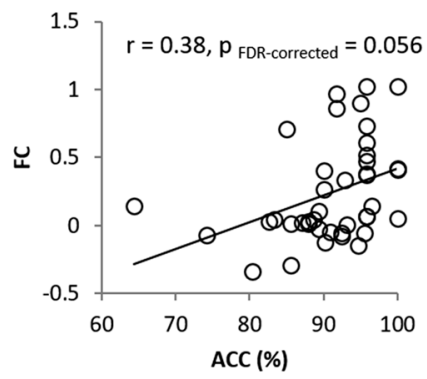

I.vATL- r.vATL

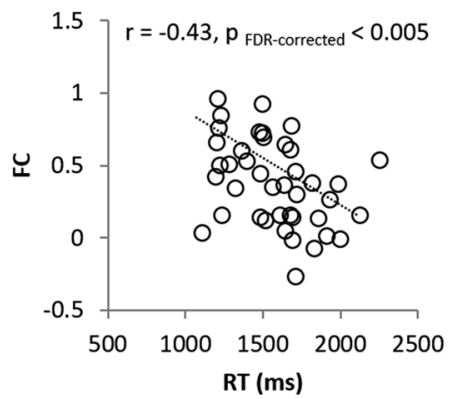

I.vATL- I.IFG (p. Tri)

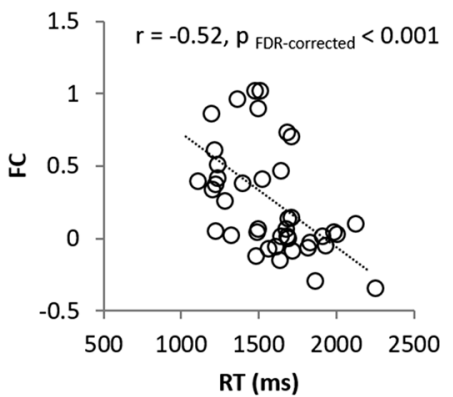

I.SN

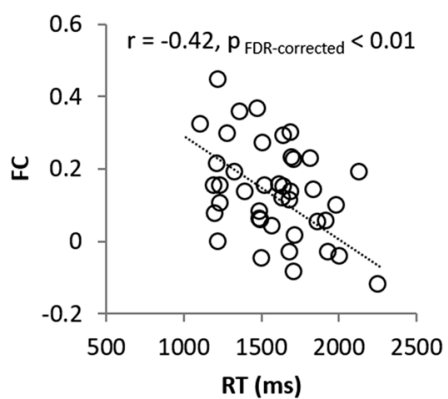

\section{B Hard semantic condition}

I.vATL-r.vATL

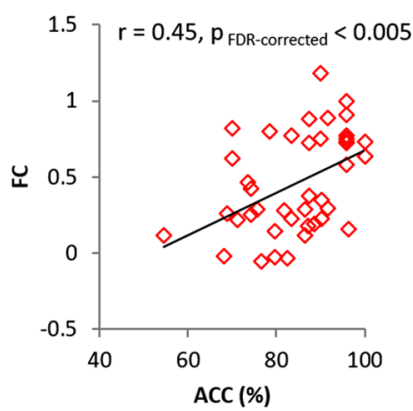

I.vATL-I.IFG (p. Tri)

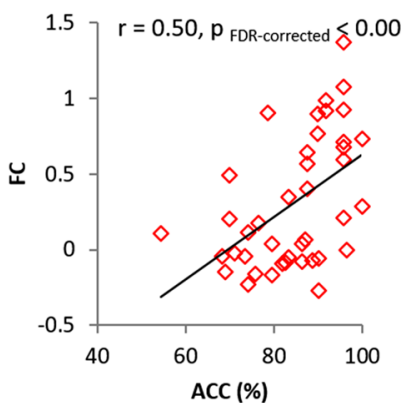

I.vATL-I.IFG (p. Orb)

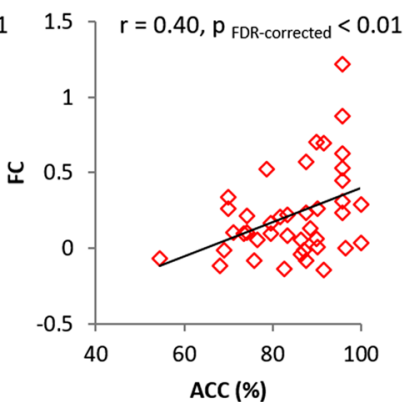

r.vATL-r.IFG (p. Orb)

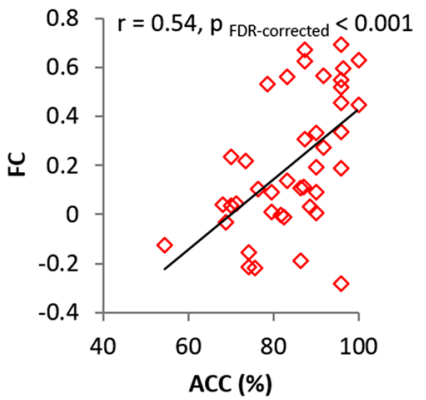

Fig. 6 FC-behavioural correlations. a Easy semantic condition. Black circle represent individual's performance during the easy semantic condition. b Hard semantic condition. Red diamond indicates each individual's performance (accuracy) during the hard semantic condition

vATL, left vATL-left IFG, and left SN connectivity $\left(p_{\text {FDR-corrected }}<0.05\right)($ Fig. 6a). In the hard semantic condition, there were significant correlations between accuracy and the interhemispheric vATL connectivity (left-right vATL) and between accuracy and the vATL-IFG [p. orb, p. tri] connectivity in both hemispheres $\left(p_{\text {DR-corrected }}<0.05\right)$ (Fig. 6b). We did not run the analysis for the RT in the hard semantic condition, as there was no difficulty effect in the combined dataset $(\mathrm{SM}+\mathrm{RM})$. Overall, individuals with stronger FC between the semantic regions performed the semantic task better (i.e., faster RT and/or higher accuracy). The same analyses were performed in each dataset, split according to modality, and the results are summarised in Fig. S8.

\section{Discussion}

The overarching aim of this study was to begin the examination of the mechanisms that make higher cognitive systems well engineered and thus resilient to variable performance demands, perturbation and minor damage. Beyond investigations of executive function (where demand variations are central to the experimental design), other domains of 
higher cognition are often examined at a single performance demand level in each study (though at different levels across studies) whilst other parameters of interest are manipulated. Yet the question of how resilience is achieved in higher cognitive systems is important both for cognitive and clinical neuroscience. In this study, we explored the intrinsic neural dynamics of the semantic network in healthy participants and how the system alters to be resilient to changes in performance demand. The results directly paralleled those found in previous investigations of the semantic network after neurological damage (Bi et al. 2011; Lambon Ralph et al. 2010a) or TMS perturbation (Pobric et al. 2007; Binney and Lambon Ralph 2015; Jung and Lambon Ralph 2016) suggesting that the well-engineered neurocognitive network for semantic cognition has intrinsic mechanisms that make it resilience to changing performance demands, which are also engaged when the system is partially compromised. In summary, we found that semantically demanding conditions upregulated the left-lateralized semantic regions including the vATL, IFG and pMTG as well as other regions in domain general networks. More specifically, there was increased activation in the bilateral ATL supporting the notion that semantic representation is inherently bilateral in nature (Lambon Ralph et al. 2017; Rice et al. 2015, 2018). Functional connectivity between these regions (interhemispheric vATL connectivity and the vATL-PFC) was also increased. Importantly, this strengthened connectivity was associated with better semantic task performance. Our results suggest that semantic cognition is founded on a flexible, dynamic system making itself resilient to internal (e.g., system damage) and external changes (e.g., task demands).

Importantly, these intrinsic resilience-related mechanisms were observed in both the domain-specific representational system and the parallel executive control networks. We observed dynamic changes in the semantic representational system: semantically demanding conditions increased the regional activity in the bilateral vATL and functional connectivity between them, which correlated with semantic performance. Note these same demand-related areas were also found in the easy condition if the statistical threshold was reduced. This fits with the notion that there is an intrinsic broader network that can support function but, to save energy, its level of activation is titrated against performance demand-'variable neuro-displacement' (Stefaniak et al. 2020). This mechanism is analogous to variable displacement in combustion engines where cylinder function is down-regulated or switched off to save energy but are re-engaged when increased performance is needed (Manring and Johnson 1996). The bilateral nature of ATLreliant semantic representation could, itself be crucial for its resilient, well-engineered characteristics. Past studies of healthy participants after inhibitory ATL rTMS, patients with resection for temporal lobe epilepsy, and comparative neurosurgical investigations have shown that bilateral lesions are required for substantial, chronic semantic impairments (i.e., breaking the resilience of the system) and that after unilateral stimulation or resection, there are compensatory upregulations of activation in contralateral regions, peridamage areas and increased positive transcallosal functional connectivity (Binney and Lambon Ralph 2015; Jung and Lambon Ralph 2016; Rice et al. 2018; Kluver and Bucy 1939; Terzian and Ore 1955). The resilience that follows from bilateral neural systems has also been formally demonstrated and explored through implemented neurocomputational models of bilateral semantic representation. These show that a bilaterally supported representation system is much more resilient to unilateral damage and has greater capacity for experience-dependent plasticity-related recovery (Schapiro et al. 2013). These bilaterally supported functional networks in response to increased demands have been reported in other cognitive domains (Hartwigsen 2018; Hartwigsen et al. 2013).

As well as these dynamic changes in the domain-specific network of interest (e.g., semantic representation), we also observed upregulations in the parallel executive-control networks and their connectivity to the domain-specific representations. The assistance from the executive systems, again, reflects its inherent function in the healthy system, i.e., when processing is difficult or the representations need to be adjusted then the executive systems kick in (Badre et al. 2005; Badre and Wagner 2002; Noonan et al. 2013). Here, the demanding semantic condition upregulated the IFG and pMTG plus increased functional connectivity between them as well as between the vATL and IFG. Importantly, individuals with stronger vATL-IFG connectivity performed the semantic task better in both easy and hard conditions.

These types of changes and their associated explanations, directly mirror previous patient (Bi et al. 2011; Lambon Ralph et al. 2010a) and TMS explorations (Binney and Lambon Ralph 2015; Jung and Lambon Ralph 2016). This would seem to imply that the dynamic changes observed following brain damage or perturbation may not reflect de novo mechanisms that are triggered by brain damage but rather reflect the inherent mechanisms found in a well-engineered, resilient cognitive system. Turning to the engine analogy again, this is the same as running the engine at higher demand levels (and not downregulating) when a part of the engine is compromised. After brain damage or TMS perturbation in the semantic system, the upregulation of the remaining representational and executive-control regions was observed and associated with the remaining semantic ability (Backes et al. 2005; Maccotta et al. 2007; Binney and Lambon Ralph 2015; Jung and Lambon Ralph 2016). A recent TMS-fMRI study demonstrated the increased interhemispheric vATL-connectivity in semantic processing after perturbing the left vATL(Jung 
and Lambon Ralph 2016). Strikingly, we showed the same results in the healthy system by increasing task demand, suggesting that the same mechanism may underpin such changes in patients and TMS investigations.

These results also have potential implications for investigations of recovery in patients. Typically, patients and controls are asked to complete exactly the same task in the scanner, with the task adjusted so that patients are able to perform reasonably well within the scanner. Differences between patients and controls are assumed to reflect newly engaged regions/mechanisms and that these are the basis of recovery or compensation. The current results suggest, however, that easier versions of the same task may under-estimate the entire cognitive network and, in fact, the patients' results might simply reflect the function of the remaining (full-engaged) cognitive network. In keeping with this hypothesis, recent patient studies have started to compare patient data against control data collected at two levels of demand (Rice et al. 2018; Sharp et al. 2004; Brownsett et al. 2014). For example, Brownsett et al. (2014) showed that the upregulated regions observed in post-stroke aphasic patients whilst listening to clear speech aligned with regions that heathy controls upregulated when listening to degraded speech. Likewise, epilepsy patients with temporal lobe resection (either left or right) also showed similar pattern of upregulation in the intact ATL and PFC to that of healthy controls when given a shortened response time window (Rice et al. 2018). These results provide strong evidence that the compensatory functional alterations in the impaired brain might reflect intrinsic mechanisms of a wellengineered semantic system.

Finally, we note that the focus of the current study was limited to the semantic system. The purpose of the current study was to explore the neural mechanism resilient to functional demands and stresses in the healthy population. Future studies will be able to assess whether these results hold across other cognitive domains, including testing the hypothesis that resilience in higher cognitive systems reflects a combination of domain-general (executive) and domainspecific networks. As well as revealing important insights about the constitution of well-engineered healthy systems, these studies should elicit potentially important insights about the neural mechanisms that support compensatory dynamic changes after brain damage or surgery. Secondly, to test the generality of the resilience-related responses, we used different types of challenge and stimulus modality in this study. Whilst the overall findings were similar across them, there were some differences between the task modality (word vs. picture) and the difficulty manipulations (SM vs. RM). Future studies including a greater number of participants in each condition, are needed to investigate these and other types of difficulty/demand-related manipulations and to explore what processes or representations they reflect.
Supplementary Information The online version contains supplementary material available at https://doi.org/10.1007/s00429-021-02272-1.

Funding This research was supported by a Beacon Anne McLaren Research Fellowship (University of Nottingham) to JJ and an Advanced ERC award (GAP: 670428-BRAIN2MIND_NEUROCOMP), MRC programme grant (MR/R023883/1) and intramural funding (MC_ UU_00005/18) to MALR.

Data availability statement The data that support the findings of this study are available on request from the corresponding author, J.J and M.A.L.

\section{Declarations}

Conflict of interest The authors declare no conflict of interest.

Open Access This article is licensed under a Creative Commons Attribution 4.0 International License, which permits use, sharing, adaptation, distribution and reproduction in any medium or format, as long as you give appropriate credit to the original author(s) and the source, provide a link to the Creative Commons licence, and indicate if changes were made. The images or other third party material in this article are included in the article's Creative Commons licence, unless indicated otherwise in a credit line to the material. If material is not included in the article's Creative Commons licence and your intended use is not permitted by statutory regulation or exceeds the permitted use, you will need to obtain permission directly from the copyright holder. To view a copy of this licence, visit http://creativecommons.org/licenses/by/4.0/.

\section{References}

Ashburner J (2007) A fast diffeomorphic image registration algorithm. Neuroimage 38(1):95-113. https://doi.org/10.1016/j. neuroimage.2007.07.007

Backes WH, Deblaere K, Vonck K, Kessels AG, Boon P, Hofman P, Wilmink JT, Vingerhoets G, Boon PA, Achten R, Vermeulen J, Aldenkamp AP (2005) Language activation distributions revealed by fMRI in post-operative epilepsy patients: differences between left- and right-sided resections. Epilepsy Res 66(1-3):1-12. https://doi.org/10.1016/j.eplepsyres.2005.06.007

Badre D, Wagner AD (2002) Semantic retrieval, mnemonic control, and prefrontal cortex. Behav Cogn Neurosci Rev 1(3):206-218

Badre D, Poldrack RA, Pare-Blagoev EJ, Insler RZ, Wagner AD (2005) Dissociable controlled retrieval and generalized selection mechanisms in ventrolateral prefrontal cortex. Neuron 47(6):907-918. https://doi.org/10.1016/j.neuron.2005.07.023

Behzadi Y, Restom K, Liau J, Liu TT (2007) A component based noise correction method (CompCor) for BOLD and perfusion based fMRI. Neuroimage 37(1):90-101. https://doi.org/10. 1016/j.neuroimage.2007.04.042

Bi Y, Wei T, Wu C, Han Z, Jiang T, Caramazza A (2011) The role of the left anterior temporal lobe in language processing revisited: evidence from an individual with ATL resection. Cortex 47(5):575-587. https://doi.org/10.1016/j.cortex.2009.12.002

Billingsley RL, McAndrews MP, Crawley AP, Mikulis DJ (2001) Functional MRI of phonological and semantic processing in temporal lobe epilepsy. Brain 124(Pt 6):1218-1227

Binder JR, Desai RH, Graves WW, Conant LL (2009) Where is the semantic system? A critical review and meta-analysis of 120 
functional neuroimaging studies. Cereb Cortex 19(12):27672796. https://doi.org/10.1093/cercor/bhp055

Binney RJ, Lambon Ralph MA (2015) Using a combination of fMRI and anterior temporal lobe rTMS to measure intrinsic and induced activation changes across the semantic cognition network. Neuropsychologia 76:170-181. https://doi.org/10. 1016/j.neuropsychologia.2014.11.009

Binney RJ, Embleton KV, Jefferies E, Parker GJ, Ralph MA (2010) The ventral and inferolateral aspects of the anterior temporal lobe are crucial in semantic memory: evidence from a novel direct comparison of distortion-corrected fMRI, rTMS, and semantic dementia. Cereb Cortex 20(11):2728-2738. https:// doi.org/10.1093/cercor/bhq019

Bozeat S, Lambon Ralph MA, Patterson K, Garrard P, Hodges JR (2000) Non-verbal semantic impairment in semantic dementia. Neuropsychologia 38(9):1207-1215

Brown SSE (1888) An investigation into the functions of the occipital and temporal lobes of the monkey's brain. Philos Trans R Soc Lond B Biol Sci 179:303-327. https://doi.org/10.1098/rstb.1888. 0011

Brownsett SL, Warren JE, Geranmayeh F, Woodhead Z, Leech R, Wise RJ (2014) Cognitive control and its impact on recovery from aphasic stroke. Brain 137(Pt 1):242-254. https://doi.org/10.1093/brain/ awt 289

Chan D, Fox NC, Scahill RI, Crum WR, Whitwell JL, Leschziner G, Rossor AM, Stevens JM, Cipolotti L, Rossor MN (2001) Patterns of temporal lobe atrophy in semantic dementia and Alzheimer's disease. Ann Neurol 49(4):433-442

Clarke A, Taylor KI, Tyler LK (2011) The evolution of meaning: spatio-temporal dynamics of visual object recognition. J Cogn Neurosci 23(8):1887-1899. https://doi.org/10.1162/jocn.2010.21544

Coccia M, Bartolini M, Luzzi S, Provinciali L, Ralph MA (2004) Semantic memory is an amodal, dynamic system: evidence from the interaction of naming and object use in semantic dementia. Cogn Neuropsychol 21(5):513-527. https://doi.org/10.1080/ 02643290342000113

Deco G, Tononi G, Boly M, Kringelbach ML (2015) Rethinking segregation and integration: contributions of whole-brain modelling. Nat Rev Neurosci 16(7):430-439. https://doi.org/10.1038/nrn3963

Goni J, van den Heuvel MP, Avena-Koenigsberger A, Velez de Mendizabal N, Betzel RF, Griffa A, Hagmann P, CorominasMurtra B, Thiran JP, Sporns O (2014) Resting-brain functional connectivity predicted by analytic measures of network communication. Proc Natl Acad Sci USA 111(2):833-838. https://doi. org/10.1073/pnas.1315529111

Halai AD, Welbourne SR, Embleton K, Parkes LM (2014) A comparison of dual gradient-echo and spin-echo fMRI of the inferior temporal lobe. Hum Brain Mapp 35(8):4118-4128. https://doi. org $/ 10.1002 / \mathrm{hbm} .22463$

Hartwigsen G (2018) Flexible redistribution in cognitive networks. Trends Cogn Sci 22(8):687-698. https://doi.org/10.1016/j.tics. 2018.05.008

Hartwigsen G, Saur D, Price CJ, Ulmer S, Baumgaertner A, Siebner HR (2013) Perturbation of the left inferior frontal gyrus triggers adaptive plasticity in the right homologous area during speech production. Proc Natl Acad Sci USA 110(41):16402-16407. https://doi.org/10.1073/pnas.1310190110

Heiss WD, Kessler J, Thiel A, Ghaemi M, Karbe H (1999) Differential capacity of left and right hemispheric areas for compensation of poststroke aphasia. Ann Neurol 45(4):430-438. https://doi.org/ 10.1002/1531-8249(199904)45:4\%3c430::aid-ana3\%3e3.0.co;2-p

Hoffman P, Binney RJ, Lambon Ralph MA (2015) Differing contributions of inferior prefrontal and anterior temporal cortex to concrete and abstract conceptual knowledge. Cortex 63:250-266. https://doi.org/10.1016/j.cortex.2014.09.001
Jefferies E (2013) The neural basis of semantic cognition: converging evidence from neuropsychology, neuroimaging and TMS. Cortex 49(3):611-625. https://doi.org/10.1016/j.cortex.2012.10.008

Jefferies E, Lambon Ralph MA (2006) Semantic impairment in stroke aphasia versus semantic dementia: a case-series comparison. Brain 129(Pt 8):2132-2147. https://doi.org/10.1093/brain/awl153

Jung J, Lambon Ralph MA (2016) Mapping the dynamic network interactions underpinning cognition: a cTBS-fMRI study of the flexible adaptive neural system for semantics. Cereb Cortex 26(8):3580-3590. https://doi.org/10.1093/cercor/bhw149

Jung J, Cloutman LL, Binney RJ, Lambon Ralph MA (2016) The structural connectivity of higher order association cortices reflects human functional brain networks. Cortex. https://doi.org/10. 1016/j.cortex.2016.08.011

Kluver H, Bucy PC (1939) Preliminary analysis of functions of the temporal lobes in monkeys. Arch Neuro Psychiatr 42(6):979-1000

Lambon Ralph MA (2014) Neurocognitive insights on conceptual knowledge and its breakdown. Philos Trans R Soc Lond B Biol Sci 369(1634):20120392. https://doi.org/10.1098/rstb.2012.0392

Lambon Ralph MA, Cipolotti L, Manes F, Patterson K (2010a) Taking both sides: do unilateral anterior temporal lobe lesions disrupt semantic memory? Brain 133(11):3243-3255. https://doi.org/10. 1093/brain/awq264

Lambon Ralph MA, Sage K, Jones RW, Mayberry EJ (2010b) Coherent concepts are computed in the anterior temporal lobes. Proc Natl Acad Sci USA 107(6):2717-2722. https://doi.org/10.1073/ pnas.0907307107

Lambon Ralph MA, Ehsan S, Baker GA, Rogers TT (2012) Semantic memory is impaired in patients with unilateral anterior temporal lobe resection for temporal lobe epilepsy. Brain 135:242-258. https://doi.org/10.1093/brain/awr325

Lambon Ralph MA, Jefferies E, Patterson K, Rogers TT (2017) The neural and computational bases of semantic cognition. Nat Rev Neurosci 18(1):42-55. https://doi.org/10.1038/nrn.2016.150

Maccotta L, Buckner RL, Gilliam FG, Ojemann JG (2007) Changing frontal contributions to memory before and after medial temporal lobectomy. Cereb Cortex 17(2):443-456. https://doi.org/10.1093/ cercor/bhj161

Manring ND, Johnson RE (1996) Modeling and designing a variable-displacement open-loop pump. J Dyn Syst Trans Asme 118(2):267-271. https://doi.org/10.1115/1.2802313

Murray LL (2012) Attention and other cognitive deficits in aphasia: presence and relation to language and communication measures. Am J Speech Lang Pathol 21(2):S51-64. https://doi.org/10.1044/ 1058-0360(2012/11-0067)

Nee DE, Brown JW, Askren MK, Berman MG, Demiralp E, Krawitz A, Jonides J (2013) A meta-analysis of executive components of working memory. Cereb Cortex 23(2):264-282. https://doi.org/ 10.1093/cercor/bhs007

Noonan KA, Jefferies E, Visser M, Lambon Ralph MA (2013) Going beyond inferior prefrontal involvement in semantic control: evidence for the additional contribution of dorsal angular gyrus and posterior middle temporal cortex. J Cogn Neurosci 25(11):18241850. https://doi.org/10.1162/jocn_a_00442

Noppeney U, Phillips J, Price C (2004) The neural areas that control the retrieval and selection of semantics. Neuropsychologia 42(9):1269-1280. https://doi.org/10.1016/j.neuropsychologia. 2003.12.014

Noppeney U, Price CJ, Duncan JS, Koepp MJ (2005) Reading skills after left anterior temporal lobe resection: an fMRI study. Brain 128(Pt 6):1377-1385. https://doi.org/10.1093/brain/awh414

O'Reilly JX, Woolrich MW, Behrens TE, Smith SM, Johansen-Berg H (2012) Tools of the trade: psychophysiological interactions and functional connectivity. Soc Cogn Affect Neurosci 7(5):604-609. https://doi.org/10.1093/scan/nss055 
Patterson K, Nestor PJ, Rogers TT (2007) Where do you know what you know? The representation of semantic knowledge in the human brain. Nat Rev Neurosci 8(12):976-987. https://doi.org/ $10.1038 / \mathrm{nrn} 2277$

Paus T (2005) Inferring causality in brain images: a perturbation approach. Philos Trans R Soc Lond B Biol Sci 360(1457):1109_ 1114. https://doi.org/10.1098/rstb.2005.1652

Peelen MV, Caramazza A (2012) Conceptual object representations in human anterior temporal cortex. J Neurosci 32(45):15728-15736. https://doi.org/10.1523/JNEUROSCI.1953-12.2012

Pobric G, Jefferies E, Ralph MA (2007) Anterior temporal lobes mediate semantic representation: mimicking semantic dementia by using rTMS in normal participants. Proc Natl Acad Sci USA 104(50):20137-20141. https://doi.org/10.1073/pnas.0707383104

Poch C, Garrido MI, Igoa JM, Belinchon M, Garcia-Morales I, Campo P (2015) Time-varying effective connectivity during visual object naming as a function of semantic demands. J Neurosci 35(23):8768-8776. https://doi.org/10.1523/JNEUROSCI.488814.2015

Rice GE, Lambon Ralph MA, Hoffman P (2015) The roles of left versus right anterior temporal lobes in conceptual knowledge: an ALE meta-analysis of 97 functional neuroimaging studies. Cereb Cortex 25(11):4374-4391. https://doi.org/10.1093/cercor/bhv024

Rice GE, Caswell H, Moore P, Lambon Ralph MA, Hoffman P (2018) Revealing the dynamic modulations that underpin a resilient neural network for semantic cognition: an fMRI investigation in patients with anterior temporal lobe resection. Cereb Cortex 28(8):3004-3016. https://doi.org/10.1093/cercor/bhy116

Rogers TT, Patterson K, Jefferies E, Ralph MA (2015) Disorders of representation and control in semantic cognition: effects of familiarity, typicality, and specificity. Neuropsychologia 76:220-239. https://doi.org/10.1016/j.neuropsychologia.2015.04.015

Saur D, Lange R, Baumgaertner A, Schraknepper V, Willmes K, Rijntjes M, Weiller C (2006) Dynamics of language reorganization after stroke. Brain 129(Pt 6):1371-1384. https://doi.org/10. 1093/brain/awl090

Schapiro AC, McClelland JL, Welbourne SR, Rogers TT, Lambon Ralph MA (2013) Why bilateral damage is worse than unilateral damage to the brain. J Cogn Neurosci 25(12):2107-2123. https:// doi.org/10.1162/jocn_a_00441

Sharp DJ, Scott SK, Wise RJ (2004) Retrieving meaning after temporal lobe infarction: the role of the basal language area. Ann Neurol 56(6):836-846. https://doi.org/10.1002/ana.20294

Spreng RN, Sepulcre J, Turner GR, Stevens WD, Schacter DL (2013) Intrinsic architecture underlying the relations among the default, dorsal attention, and frontoparietal control networks of the human brain. J Cogn Neurosci 25(1):74-86. https://doi.org/10.1162/ jocn_a_00281

Stefaniak JD, Halai AD, Lambon Ralph MA (2020) The neural and neurocomputational bases of recovery from post-stroke aphasia. Nat Rev Neurol 16(1):43-55. https://doi.org/10.1038/ s41582-019-0282-1

Szaflarski JP, Allendorfer JB, Banks C, Vannest J, Holland SK (2013) Recovered vs. not-recovered from post-stroke aphasia: the contributions from the dominant and non-dominant hemispheres. Restor Neurol Neurosci 31(4):347-360. https://doi.org/10.3233/ RNN-120267

Terzian H, Ore GD (1955) Syndrome of Kluver and Bucy; reproduced in man by bilateral removal of the temporal lobes. Neurology 5(6):373-380

Thompson-Schill SL, D'Esposito M, Aguirre GK, Farah MJ (1997) Role of left inferior prefrontal cortex in retrieval of semantic knowledge: a reevaluation. Proc Natl Acad Sci USA 94(26):14792-14797

van Oers CA, Vink M, van Zandvoort MJ, van der Worp HB, de Haan EH, Kappelle LJ, Ramsey NF, Dijkhuizen RM (2010) Contribution of the left and right inferior frontal gyrus in recovery from aphasia. A functional MRI study in stroke patients with preserved hemodynamic responsiveness. Neuroimage 49(1):885-893. https://doi.org/10.1016/j.neuroimage.2009.08.057

Wagner AD, Pare-Blagoev EJ, Clark J, Poldrack RA (2001) Recovering meaning: left prefrontal cortex guides controlled semantic retrieval. Neuron 31(2):329-338. https://doi.org/10.1016/S08966273(01)00359-2

Warburton E, Price CJ, Swinburn K, Wise RJ (1999) Mechanisms of recovery from aphasia: evidence from positron emission tomography studies. J Neurol Neurosurg Psychiatry 66(2):155-161. https://doi.org/10.1136/jnnp.66.2.155

Woolgar A, Bor D, Duncan J (2013) Global increase in task-related fronto-parietal activity after focal frontal lobe lesion. J Cogn Neurosci 25(9):1542-1552. https://doi.org/10.1162/jocn_a_00432

Publisher's Note Springer Nature remains neutral with regard to jurisdictional claims in published maps and institutional affiliations. 